\title{
Conservation Genomics of Urban Populations of Streamside Salamander (Ambystoma
} barbouri)

N. Wade Hubbs ${ }^{\&}$, Carla R. Hurt ${ }^{1 \&^{*}}$, John Niedzwiecki², Brian Leckie ${ }^{1}$ and David Withers ${ }^{3}$

${ }^{1}$ Department of Biology, Tennessee Technological University, Cookeville, TN

${ }^{2}$ Belmont University, Nashville TN

${ }^{3}$ Tennessee Department of Environment and Conservation, Nashville, TN

*Correspondence: Carla Hurt

E-mail: churt@tntech.edu

\&Authors contributed equally to this work 


\section{Abstract}

3 barbouri) are in decline as their distribution lies mostly within rapidly developing areas in the

4 Nashville Basin. Information regarding the partitioning of genetic variation among populations

5 of $A$. barbouri, and the taxonomic status of these populations relative to northern populations

6 and their congener, the smallmouth salamander (A. texanum), have important implications for

7 management and conservation of this species. Here we combined mitochondrial sequencing and

8 genome-wide single nucleotide polymorphism (SNP) data generated using Genotyping-by-

9 Sequencing (GBS) to investigate patterns of genetic variation within Tennessee populations of

10 A. barbouri, to assess their relationship to populations in Kentucky, and to examine their

11 phylogenetic relationship to the closely related $A$. texanum. Results from phylogenetic

12 reconstructions reveal a complex history of Tennessee A. barbouri populations with regards to

13 northern populations, unisexual A. barbouri, and A. texanum. Patterns of mitochondrial

14 sequence variation suggest that $A$. barbouri may have originated within Tennessee and expanded

15 north multiple times into Kentucky, Ohio, Indiana and West Virginia. Phylogenetic

16 reconstructions based on genome-wide SNP data contradict results based on mitochondrial DNA

17 and correspond to geographic and taxonomic boundaries. Variation in allele frequencies at SNP

18 genotypes, as identified by multivariate analyses and Bayesian assignment tests, identified three

19 evolutionary significant units (ESUs) for A. barbouri within the state of Tennessee. Collectively,

20 these results emphasize the need for prioritizing conservation needs of Tennessee populations of

21 A. barbouri to ensure the long-term persistence of this species. 
Genetic variation, population structure and demographic history are increasingly

recognized as important factors for the design of effective conservation strategies (Geist 2010).

Streamside salamander (Ambystoma barbouri, Fig. 1) populations in Middle Tennessee are declining due to rapid urbanization in and around the Nashville Basin and as a result, were reclassified from "deemed in need of management" to state endangered by the Tennessee Wildlife Resources Agency (TWRA) (Withers et al. 2009, TWRA 2018, Anderson et al. 2014). Population fragmentation and loss of genetic variation that inevitably accompany loss of habitat threaten the long-term adaptive potential and persistence of $A$. barbouri, but can be mitigated by management actions aimed at maintaining genetic diversity. Information regarding $A$. barbouri's taxonomy, population structure and patterns of genetic variation are currently needed to prioritize conservation needs and to efficiently allocate management resources. Genomic tools are increasingly being used to improve recovery and management planning in at-risk salamander species by informing taxonomic relationships, demographic histories, and biologically meaningful units of conservation that will preserve genetic diversity and the long-term adaptive potential of species.

Taxonomic uncertainties surrounding the evolutionary relationship between $A$. barbouri and its sister-species $A$. texanum (smallmouthed salamander) are a concern for precisely identifying targets of conservation. These two species are nearly indistinguishable based on external morphological features alone and were previously considered to be conspecific. They can be differentiated using scanning electron microscopy on the number and shape of maxillary and premaxillary teeth, life-history traits, and choice of breeding environment (Niemiller et al. 2009, Kraus \& Petranka 1989). In addition to their morphological ambiguity, phylogenetic 
reconstructions using mitochondrial and nuclear sequence data also produced conflicting results

47 regarding the relationship between $A$. texanum and $A$. barbouri (Niedzwiecki 2005).

48 Reconstructions based on mitochondrial sequences show that A. texanum and A. barbouri are

49 not reciprocally monophyletic; $A$. texanum is recovered as a clade nested within $A$. barbouri

50 from Tennessee, suggesting that pond-breeding $A$. texanum were more recently derived from a

51 stream-breeding A. barbouri ancestor from Central Tennessee. However, reconstructions based

52 on two nuclear gene sequences resulted in reciprocally monophyletic clades for the two species,

53 consistent with a history where $A$. barbouri and $A$. texanum are older independent lineages

54 derived from a shared common ancestor. Additional sequence data are needed to resolve

55 unanswered phylogenetic questions as these earlier reconstructions were based on a limited

56 number of molecular markers (two nuclear and one mitochondrial gene) and representative

57 outgroups.

Mitochondrial evidence also informs on the origins of unisexual Ambystomatid populations that are common in the Great Lakes region of North America. Unisexual

60

61

62

63
Ambystomatids exhibit a unique reproductive mode whereby male sperm activates egg development, but contributes variable amounts of nuclear genetic material depending on compatibility with unrelated cytoplasmic DNA (termed kleptogenesis). While the nuclear genome unisexuals is a mixture of different Ambystomatid species, all known mitochondrial haplotypes, across their range, nest within A. barbouri. Mitochondrial haplotypes are most closely related to mitochondrial haplotypes found south of the Ohio River, and southwest of the Kentucky river (Bogart et al. 2007); however, the relationship of Tennessee $A$. barbouri to unisexual Ambystomatids has not been explored. 
At a finer scale, uncertainties also exist regarding the relationship among isolated

populations of $A$. barbouri in Tennessee and discontinuous populations in Kentucky, Indiana, mitochondrial sequence data (913 bp) from the D-loop and an adjacent intergenic spacer suggest that Tennessee $A$. barbouri populations are both ecologically and genetically distinct from more northern populations (Eastman et al. 2009). At hatching, A. barbouri from Tennessee were

74 smaller and less developed than individuals from Kentucky (Niedzwiecki 2005). Also,

75 laboratory behavioral assays show that Tennessee $A$. barbouri were similar to western Kentucky

76 individuals, but less active than individuals from northern populations (Niedzwiecki 2005).

77 These differences were supported by genetic data; phylogenetic reconstructions recovered

78 mitochondrial haplotypes from Tennessee as monophyletic and basal to haplotypes from

79 Kentucky, Ohio, and West Virginia (Eastman et al. 2009). These results support an early

80 divergence of Tennessee populations and raise questions regarding the geographic origin of $A$. barbouri. However, conclusions from this study were limited as these reconstructions are based on a single mitochondrial gene from only three individuals from a few populations within a kilometer of each other in Rutherford County. in decline, and the accompanying loss of genetic variation in small populations further threatens

86 the long-term persistence of this species (Niemiller et al. 2006). Maintenance of genetic

87 variation is a fundamental priority for conservation planning and requires information regarding

88 the partitioning of genetic variation within and between isolated populations. Data on patterns of genetic variation in Tennessee populations of $A$. barbouri are limited to a handful of 
91 Niedzweicki 2005). Genome-wide surveys of genetic variation from fine-scale population

92 sampling across the state are critical for assessing genetic variation within populations, establishing units of conservation and maintaining historical patterns of gene flow between

94 populations to improve the outcome of recovery efforts (Shaffer et al. 1996).

Here we investigate patterns of genetic variation within and among populations of

96 Tennessee $A$. barbouri as well as the taxonomic relationship between $A$. barbouri and $A$.

97 texanum in order to prioritize conservation needs and inform management practices aimed at maximizing long-term persistence of this species. Mitochondrial sequence data and genome wide SNP genotypes were used to (1) investigate the phylogenetic relationship between $A$. barbouri and $A$. texanum, (2) review the taxonomic relationship of disjunct populations of $A$.

101 barbouri in Tennessee relative to northern populations, (3) evaluate patterns of genetic 102 differentiation between geographically isolated populations of A. barbouri within the state of

103 Tennessee and (4) estimate within-population genetic variation and examine demographic history of Tennessee $A$. barbouri. Taxonomic relationships, units of conservation, and geographic partitioning of genetic variation are discussed in the context of establishing conservation priorities and designing effective management strategies for this species.

\section{Methods}

Tissue Collection and DNA Extraction.

Tissue samples of $A$. barbouri in the form of adult tail clips, eggs, and whole larvae were

110 obtained from field surveys or from collaborators (Fig. 2, Table 1). Historical and predicted sites

111 of $A$. barbouri were surveyed from January 2018 to March 2018 and again from November 2018

112 to March 2019, coinciding with timing of oviposition as reported by Niemiller et al. (2009). At

113 each site, $A$. barbouri adults and eggs were collected by turning over cover objects within and 
near seasonal streams. Additionally, pools were searched for free-swimming larvae; only one larva was sampled from any single pool to avoid sampling related individuals from the same clutch. A total of 235 individuals were included for genetic analysis. Samples included 225 individuals from 13 populations of $A$. barbouri in Tennessee spanning six counties in the Nashville Basin: Bedford County (B6), Davidson County (D3), Rutherford County (R1, R7, and R9), Sumner County (S2, S5, S7, S8), Wilson County (W1, W3, and W4), and Williamson County (Wil2). A total of five $A$. texanum individuals were sampled; these included four individuals from (Arnold Airforce Base, AAFB) and one from Craighead Co., Arkansas (AKT1). Outgroups included two A. mabeei (North Carolina), two A. maculatum (AAFB), and one $A$. talpoideum (AAFB).

DNA was extracted from tail clips, eggs, and larvae using the EZNA Tissue DNA Mini kit (OMEGA BIO-TEK) following the manufacturer's protocol, except that DNA was eluted in water. Approximately $1200 \mathrm{bp}$ of the mitochondrial D-loop was targeted for PCR amplification using primers developed by Shaffer and McKnight (1996; Table 2). The mitochondrial D-loop has been shown to be informative for evaluating population structure and species relationships within the genus Ambystoma (Bogart et al. 2007; Charney et al. 2014; Church et al.; 2003; Shaffer \& McKnight 1996; Zamudio \& Savage 2003). Conditions for polymerase chain reaction (PCR) were as follows: initial denaturation step of 2 minutes at $95^{\circ} \mathrm{C}$, followed by 35 cycles of $15 \mathrm{~s}$ at $95^{\circ} \mathrm{C}, 15 \mathrm{~s}$ at $53^{\circ} \mathrm{C}$, and $90 \mathrm{~s}$ at $72^{\circ} \mathrm{C}$. This program ended with a final extension of $10 \mathrm{~min}$. at $72^{\circ} \mathrm{C}$. Amplified PCR product was cleaned prior to cycle sequencing by exonuclease I/shrimp alkaline phosphatase (New England Biolabs) and used for bi-directional Sanger sequencing on an ABI 3730 automated sequencer (MCLAB). Sequence chromatograms were imported and visualized using SEQUENCHER 5.2 (Gene Codes Corporation). Sequences were aligned using 
137 ClustalW Multiple Alignment option (Thompson et al. 2003) as implemented in Bioedit (Hall

138 1999).

Phylogenetic Reconstructions for Mitochondrial Haplotypes

Phylogenetic reconstructions were estimated for all unique mitochondrial D-loop

141 haplotypes and an additional 53 A. barbouri, 83 unisexual Ambystomatids and 27 A. texanum

142 sequences obtained from Genbank. Both maximum likelihood (ML) and Bayesian optimality

143 criteria were used for phylogenetic analyses. Maximum-likelihood analyses were performed

144 using the software RAxML (Stamatakis 2014) on the CIPRES Science Gateway (Miller et al.

145 2010) under the GTR + G model. Nodal support was estimated using rapid bootstrapping (1000

146 replicates). Bayesian phylogenetic reconstructions were performed using MrBayes 3.2.1

147 (Huelsenbeck \& Ronquist 2001) also on the CIPRES Science Gateway. The best model of

148 substitution was selected by Modeltest (Posada \& Crandall 1998), implemented in MEGA X

149 (Kumar et al. 2016) using Bayesian information criterion (BIC). The Markov chain Monte Carlo

150 (MCMC) algorithm ran for 10,000,000 generations, sampling every 1,000 generations. Two

151 independent runs were performed and the resulting trees were combined after the deletion of a

152 burnin (25\%). A majority-rule consensus tree was generated and nodal support was estimated by

153 posterior probabilities.

154 GBS Library Preparation and Sequencing.

A total of 227 individuals were included for GBS sequencing including 201 individuals

156 from 12 populations of $A$. barbouri in Tennessee, 17 individuals from five populations of $A$.

157 barbouri in Kentucky, five individuals from two populations of $A$. texanum, and two sampled

158 outgroups A. talpoideum $(\mathrm{N}=1)$ and $A$. mabeei $(\mathrm{N}=1$; Table 1$)$. Genotyping-by-Sequencing

159 libraries were prepared using the restriction enzyme ApeKI following the protocol of Elshire et 
al. (2011). Genomic DNA was quantified using Quant-iT Picogreen dsDNA Assay Kit (Thermo Fisher Scientific), and all samples were standardized to between 5-6.5 ng/uL (50-65 ng total genomic DNA). Extracted DNA was digested with the restriction enzyme ApeKI. Adaptors containing PCR binding sites and individual barcodes were ligated onto digested DNA.

Barcoded DNA was pooled, and PCR was amplified using primers that bind to the ligated adaptors (see Elshire et al. 2011 for primer sequences). The resulting PCR products were cleaned using the Qiagen PCR purification kit and then cleaned again using the AxyPrep Mag PCR Clean-up kit (Axygen, Big Flats, New York, USA). The distribution of the fragment size in the PCR product was determined using an Agilent 2100 BioAnalyzer (Agilent Technologies Inc., Santa Clara, CA, USA). Barcoded libraries were sequenced using the Illumina NextSeq (Illumina Inc., San Diego, CA, USA) with a 75 bp single end read chemistry. SNP Discovery and Filtering.

The Stacks program process_radtags was used to filter and demultiplex raw reads based on barcoded sequences (Catchen 2011). We used the seven-step de novo clustering pipeline ipyrad v. 3.5 (Eaton 2014) to generate and filter SNP datasets used in downstream analyses. Quality filtering of raw sequence reads converted bases with Phred scores $<33$ to Ns, reads with more than 5 Ns were removed. Reads were clustered using a sequence similarity threshold of $90 \%$ both within and between sampled individuals, with a minimum read depth of six. Individuals with fewer than 500,000 reads were excluded from downstream analyses. Loci with observed heterozygosity $\left(\mathrm{H}_{\mathrm{o}}\right)$ greater than 0.5 were removed to filter out possible paralogs. The final SNP dataset was then filtered to remove loci deviating from Hardy-Weinberg equilibrium $(\mathrm{p}<0.05)$, loci genotyped in less than $60 \%$ of individuals, and SNPs with a minor allele frequency (MAF) less than 0.01. Only one SNP per tag was retained per locus. 
Population Genetic Variation and Effective Population Size

populations were estimated using the R-package DiveRsity v. 1.9.9 (Keenan et al. 2013).

186 Summary statistics were estimated separately for each population and included the proportion of

187 SNPs that were polymorphic within each population $(P)$, allelic richness $\left(A_{R}\right)$, and observed and 188 expected heterozygosity $\left(H_{o}\right.$ and $\left.H_{e}\right)$. Estimates of $P, A_{R}, H_{o}$, and $H_{e}$ were estimated for the entire SNP dataset and for the subset of SNPs that were polymorphic within each population. using both the linkage disequilibrium (LD) method and the heterozygote-excess method

192 (Zhdanova \& Pudovkin 2008) as implemented in the program $\mathrm{N}_{\mathrm{E}}$ Estimator v2 (Do et al. 2014).

193 Populations with sample sizes of less than 20 individuals were excluded from analyses as these 194 datasets did not provide enough signal for reliable $\mathrm{N}_{\mathrm{E}}$ estimation (Nunziata \& Weisrock 2018).

195 The minor allele frequency (MAF) parameter was set at 0.01 and $95 \%$ confidence intervals were 196 estimated using the parametric chi-squared method.

197 Population Structure.

202 reconstructions based on mitochondrial D-loop haplotypes were used to generate hypotheses

203 regarding higher-level structuring of populations. Significance of variance components was 204 determined using 1000 permutations. 
The optimal number of genetic clusters $(\mathrm{K})$ based on genomic SNPs was estimated using both a multivariate approach and a Bayesian-based assignment method. Discriminant Analysis of Principal Component (DAPC) was performed using the 'adegenet' package in $R$ (Jombart \&

208

209

210

211

212

213

214

215

216

217

218

219

220

221

222

223

224

225

226

Ahmed 2011, Jombart \& Collins 2015). The find.clusters function was first used to identify the optimal value of $K$ based on a Bayesian Information Criterion (BIC) process. The optimal

number of principal components was determined using the function a.score. The optimal $\mathrm{K}$ was then used to perform a DAPC analysis to describe the relationship between the genetic clusters and individual membership probabilities were assessed using the function compoplot. DAPC analysis was performed on three hierarchical datasets; these included 1) 12 populations of $A$. barbouri in Tennessee, 2) all sampled Tennessee and Kentucky A. barbouri populations and 3) all $A$. barbouri populations and four A. texanum individuals from AAFB.

Bayesian assignment tests were performed on Tennessee A. barbouri populations using the program STRUCTURE 2.3.4 (Pritchard et al. 2003). Kentucky A. barbouri populations were not included in assignment analyses due to small sample sizes at these sites. Values of $K$

(number of populations) ranged from 1 to 12 populations with 20 replicate runs per value of $K$; MCMC simulations were performed for a burn-in of 250,000 iterations and an additional $1,000,000$ iterations were retained for the final analysis. Results were summarized using the software package CLUMPAK (Kopelman et al. 2015). The optimal number of groups $(K)$ was determined using the Delta-K method (Evanno et al. 2005) as implemented in STRUCTURE HARVESTER v0.6.94 application (Earl \& VonHoldt 2012). The Distruct (1.1) application in CLUMPAK (Kopelman et al. 2015) was used to produce the final barplots.

Multispecies Coalescent using BPP 
A multispecies coalescent (MSC) model was used to examine taxonomic boundaries, estimate species trees, and estimate population divergence times ( $t=$ years before present $)$ as implemented in the software BPP v. 4.4 (Yang 2015). Input datasets were formatted as fulllength sequence alignments from our GBS sequencing and included all $A$. barbouri and $A$. texanum populations. Loci represented by fewer than 100 individuals were removed from the analysis. Joint species-delimitation and species-tree reconstructions were performed using the A11 model, where individuals were assigned to sampled populations. Run parameters were as follows: uniform rooted trees were used as the species model prior, the theta prior $\left(\theta=4 \mathrm{~N}_{\mathrm{E}} \mu\right)$ was assigned a gamma distribution with $\alpha=3$ and $\beta=0.04$ and the prior for tau $(\tau=\mathrm{t} \mu)$ was gamma distributed with $\alpha=3$ and $\beta=0.2$. Three independent MCMC runs were implemented, each with a burn-in of 5,000, a sample frequency of 10 and a total of 50,000 iterations. Species delimitation and species-tree topologies with the highest posterior probabilities from the A11 runs were used as input for the A00 analyses. The A00 model estimates divergence time parameters and longterm $\mathrm{N}_{\mathrm{E}}$, assuming fixed species assignments and a fixed species tree. The priors for A00 were the same as priors used for the A11 model. Three independent runs were also performed for the

242 A00 analysis, each with a burn-in of 5,000, a sample frequency of 10 and a total of 50,000 iterations. MCMC chains were pooled and absolute estimates of $\tau$ were converted to years before

244 present (YBP) in the BPPR using the genome wide mutation rate estimate for the vertebrate 245 nuclear genome where $\mu=1.21 \times 10^{-9}$ (Allio et al. 2019, Reis \& Yang 2019).

\section{Results}

Mitochondrial Sequencing. 
250 identified from the newly sequenced individuals, 39 haplotypes were identified from $A$. barbouri

251 populations sampled in Tennessee (See Supplement 1 for Genbank Accessions) and 15

252 haplotypes were identified from populations sampled in Kentucky. An additional six haplotypes

253 were sequenced from outgroups. The final alignment contained 126 variable sites (excluding

254 outgroups); of these, 120 sites were parsimony informative. No haplotypes were shared across

255 multiple populations.

256

Phylogenetic Reconstructions.

Both Bayesian and ML phylogenetic reconstructions of mitochondrial D-loop haplotypes identified six major clades across A. barbouri and A. texanum haplotypes (Fig. 3). Clade I included all haplotypes belonging to the unisexual lineage of $A$. barbouri with $100 \%$ posterior probability (pp) and 100\% bootstrap support (bs) for Bayesian and ML analysis, respectively. In

262 (population D3) formed a monophyletic clade that also includes Kentucky A. barbouri populations from southwest of the Kentucky River $(\mathrm{pp}=100, \mathrm{bs}=95 \%)$. Clade I and Clade II were recovered as sister clades in both analyses with strong support $(\mathrm{pp}=100$, bs $=99 \%)$. Clade

265 III included all $A$. texanum individuals ( $\mathrm{pp}=99, \mathrm{bs}=71)$ with the exception of a single $A$. texanum haplotype obtained from Genbank (ID EU980569, collected from Lawrence KA); this

267 sequence is recovered as basal to Clade III in both ML and Bayesian analyses. Clade IV groups with Clade III and included Tennessee A. barbouri individuals from Rutherford Co. (populations

269 R1, R7, and R9), Bedford Co. (B6) and Williamson Co.; pp =100, bs =71\%). Clade V included 270 all Kentucky individuals from populations NE of the Kentucky River, Ohio and Indiana (pp= 271 100, bs $=86 \%$ ). Finally, Clade VI is sister to Clade V and included all Tennessee A. barbouri 272 from Sumner Co. (populations S2, S5, S7, and S8), a single haplotype from Wilson population 
273 W3 and three haplotypes sequenced from Rutherford population R7 ( $\mathrm{pp}=100$, bs $=99 \%)$. On a

274 larger scale, all A. barbouri and A. texanum are part of the same well-supported monophyletic

275 group in both analyses $(\mathrm{pp}=100, \mathrm{bs}=100 \%)$, such that all $\mathrm{A}$. texanum haplotypes are nested

276 within A. barbouri.

277 GBS sequencing.

278 The average number of retained sequence reads per individual generated from

279 sequencing of GBS libraries was 5,780,366; 220 individuals were retained after removal of low

280 coverage individuals (defined as $<500,000$ total reads). A total of 1,558,844 loci were

281 recovered from the de novo assembly in ipyrad and 440,629 loci were retained after filtering.

282 Additional filtering for SNPs (i.e. filtering for low representation, HWE, MAF, and max

283 heterozygosity) recovered 1,169 SNPs for the dataset that included only Tennessee A. barbouri,

284516 SNPs in the dataset that included all sampled A. barbouri populations in Tennessee and

285 Kentucky, and 500 SNPs in the dataset that included all A. barbouri and A. texanum populations.

Much of the genetic variation within our SNP dataset was in the form of fixed differences

between populations (Table 3). The proportion of SNPs that were polymorphic within

292 (R1/R7) had the lowest estimates of $\mathrm{H}_{\mathrm{o}}$ and $\mathrm{H}_{\mathrm{e}}$, when considering only polymorphic loci and for

293 the entire SNP dataset.

294 Effective Population Size 
Estimates of effective population size $\left(N_{E}\right)$ were generated for five populations with

296

297

298

299

300

301

302

303

304

305

306

307

308

309

310

311

312

313

314

315

316

317

adequate sampling ( $N \geq 20$ ), including S7, S8, R1, R7, and D3 (Table 4). Results from LD

analyses ranged from $N_{E}=15(\mathrm{R} 1)$ to $N_{E}=108(108)$ (S8); the upper confidence interval for S8

was $\infty$, indicating low signal for this dataset. Results from the heterozygote excess method were

inconclusive $(\infty)$ for two of the five analyzed populations. Estimates from the remaining three

populations ranged from 24 (D3) to 406 (S7); however, CIs included $\infty$ for two of these

estimates (S7 and S8).

Population Structure.

Pairwise $\mathrm{F}_{\mathrm{ST}}$ estimates for $A$. barbouri populations within Tennessee were geographically structured in a hierarchical fashion (Table 5). Overall, $\mathrm{F}_{\mathrm{ST}}$ values averaged 0.375 and ranged from no differentiation $\left(\mathrm{F}_{\mathrm{ST}}=0\right)$ to highly differentiated $\left(\mathrm{F}_{\mathrm{ST}}=0.725, \mathrm{~S} 5 / \mathrm{R} 1\right)$. In general, populations north of the Cumberland River (S2, S5, S7, and S8) showed evidence of long-term

isolation from populations south of the Cumberland. Pairwise $\mathrm{F}_{\mathrm{ST}}$ estimates comparing

populations north and south of the Cumberland averaged 0.637 , while $\mathrm{F}_{\mathrm{ST}}$ estimates among

Tennessee populations north of the Cumberland averaged only 0.043 . Populations south of the

Cumberland were further structured; populations in Wilson and Davidson Counties (W1, W3,

W4, and D3) were diverged from populations further south in Williamson, Rutherford and

Bedford counties. Estimates of pairwise $\mathrm{F}_{\mathrm{ST}}$ comparing populations within Wilson/Davidson

averaged 0.121 and within Williamson, Rutherford and Bedford Counties averaged 0.070, while

between group pairwise $\mathrm{F}_{\mathrm{ST}}$ values were much higher, averaging 0.300 .

The three monophyletic clades of Tennessee A. barbouri recovered from mitochondrial

phylogenetic reconstructions were further examined using genome-wide SNP genotypes in an

AMOVA framework (Table 6). The majority of variance in SNP genotypes could be attributed 
to differences between the three clades (63.64\%). Differences between populations within clades only accounted for $2.09 \%$ of the total molecular variance. Differences between individuals accounted for the remaining $34.27 \%$ of variance in SNP genotypes.

Results of BIC for DAPC analysis suggested that three genetic clusters $(K=3)$ best represented genetic variation among populations sampled within Tennessee (Fig. 4A, Supp 2). Membership of each cluster was geographically partitioned and consisted of a northern cluster that included populations from Sumner County, a central cluster comprised of individuals from Wilson and Davidson Counties, and a southern cluster that represented Bedford, Rutherford, and Williamson County populations. Membership probabilities and DAPC plots indicated some degree of admixture between the central and southern clusters, but no admixture within the northern cluster (Supplement 2). A separate analysis that included Tennessee A. barbouri and the five sampled populations from Kentucky also resulted in three genetic clusters (Fig 4B). Kentucky populations of $A$. barbouri were isolated from populations in Tennessee. Central and southern populations were assigned to the same cluster and populations in Sumner county were assigned to the third cluster. When $A$. texanum individuals were included in a third analysis, results of the BIC analysis indicated the number of genetic clusters was five (Fig. 4C). Tennessee populations were assigned to northern, central, and southern clusters and scatter plots showed Tennessee populations to be tightly grouped with the fourth cluster that included all Kentucky populations. All A. texanum individuals formed the fifth cluster that was well separated from all $A$. barbouri individuals.

Analysis of Bayesian assignment tests for Tennessee populations of $A$. barbouri were consistent with results from DAPC analysis that suggested the presence of three geographically partitioned genetic clusters within the state (Fig. 5). The optimal value of $K$, as determined by 
341 the Delta K method, indicated four genetic clusters; however, increasing K from 2 to 3 did not

342 change population assignments. Assignment plots for $\mathrm{K}=2-3$ separated northern populations in

343 Sumner County from central and southern populations. When K was increased to four,

344 populations from Davidson and Wilson Counties were separated from southern populations in

345 Bedford, Rutherford and Williamson Counties. Increasing the value of $\mathrm{K}$ to 5 increased the level

346 of admixture, but did not result in geographically meaningful partitions.

347 Multispecies Coalescent Analyses.

A total of 988 loci were retained for the MSC analyses performed by BPP. All three A11 runs recovered the same taxonomic groups and species tree topology with the highest posterior probability. Out of 18 sampled populations, 14 populations were genetically distinct. Within

351 Tennessee, A. texanum and all $A$. barbouri populations, with the exception of R1/R7, were

352 identified as distinct groups (Fig. 6). Kentucky A. barbouri populations were split into two

353 groups; populations FC, JC, RR, and SL were grouped together and the SW populations was its 354 own genetic group. The separation of individual populations in this analysis does not indicate 355 that each population warrants recognition at the species-level as simulations have shown that BPP will split groups at the population-level when many loci are analyzed (Leach et al. 2019).

358 Tennessee clades recovered in our mitochondrial gene trees with the exception of the placement

359 of $A$. texanum. In the species tree, A. texanum was basal to all populations of $A$. barbouri in

360 Tennessee and Kentucky. The estimated divergence time (A00 analysis) between $A$. texanum and

361 A. barbouri was 1.6 million YBP. All Sumner A. barbouri formed a monophyletic group with

362 Kentucky populations as a monophyletic sister group. Divergence time estimates indicated that

363 Sumner populations and Kentucky populations shared a common ancestor $<500,000$ YBP. All 
YBP) and were further split into two groups that corresponded to clades recovered in the mitochondrial gene tree. Davidson and Wilson populations were recovered together in the first group (mitochondrial Clade II) and Rutherford, Bedford, and Williamson populations (mitochondrial Clade IV) formed the second group.

\section{Discussion}

Patterns of genomic variation and taxonomic relationships identified here have important

371 implications for developing management strategies aimed at the long-term conservation of $A$.

372 barbouri. Despite the documented decline of $A$. barbouri populations in the Nashville Basin,

373 results from GBS-derived SNP genotyping indicate that estimates of genetic variation in extant

374 populations of Tennessee $A$. barbouri are similar to SNP-based datasets examined from other

375 ambystomatids. Partitioning of genetic variation between Tennessee populations suggests that

376 hydrogeography of the Nashville Basin has shaped patterns of gene flow. Both mtDNA and

377 genomic SNP genotypes showed similar patterns with respect to population structure within the

378 state of Tennessee that should be used to inform the designation of units of conservation. Our

379 results also reveal a complex history of Tennessee $A$. barbouri with more northern populations

380 and with its sister species $A$. texanum. Despite marked differences in phylogenetic

381 reconstructions based on mtDNA sequencing and nuclear genotypes, collectively these results

382 indicate that Tennessee is genetically unique from Northern A. barbouri and A. texanum.

383 Genetic Variation within Populations.

Loss of genetic variation resulting from rapid population decline threatens the long-term 
387 adaptive genetic variation is particularly critical as these populations are faced with habitat

388 alteration by urbanization in addition to climate change (Schmidt et al. 2021). Evaluating the

389 genetic health of populations is valuable for management and conservation planning; however,

390 the interpretation of genetic variation estimates from SNP data is challenging due to the limited

391 number of comparable studies utilizing reduced-representation methods in salamanders.

392 Heterozygosity estimates obtained here are similar to estimates obtained in the handful of

393 published SNP-based genetic studies. In a recent survey of SNPs from ddRAD sequencing,

394 estimates of $\mathrm{H}_{\mathrm{O}}$ ranged from 0.165 to 0.269 for populations of the mole salamander $(A$.

395 talpoideum) and were slightly higher for populations of the marbled salamander (A. opacum; use on genetic variation in the northern two-lined salamander (Eurycea bislineata). Fusco et al. (2020) reported nearly identical estimates of $\mathrm{H}_{\mathrm{O}}$ from urban, suburban and rural salamander populations $\left(\mathrm{H}_{\mathrm{O}}=0.265,0.278\right.$ and 0.275 , respectively) and concluded that genetic variation had been maintained, despite habitat disturbance. However, estimates of heterozygosity may not be directly comparable across independent studies as filtering parameters in bioinformatic pipelines can influence population genetic summary statistics. Stringent filtering criteria may preferentially retain loci in conserved regions of the genome and downwardly bias estimates of

404 genetic variation (Huang \& Knowles 2014). There have been previous population genetic studies in A. texanum and A. barbouri populations based on microsatellite markers. Micheletti and

406 Storfer (2017) estimated genetic variation at 11 microsatellite loci in 76 populations of $A$.

407 barbouri distributed throughout Kentucky, Ohio, and Indiana; the average $\mathrm{H}_{\mathrm{e}}$ from their study

408 ranged from $0.67-0.81$, respectively. Few conclusions can be made by comparing results from 
410 known to have a much higher rate of mutation than nucleotide substitutions, increasing the

411 expected amount of allelic diversity for a given population size (Haasl \& Payseur 2011).

412 Effective population size.

413 A reduction in estimates of contemporary effective population size $\left(\mathrm{N}_{\mathrm{E}}\right)$ may also signal a

414 decline in the genetic health of at-risk populations. Our estimates of $\mathrm{N}_{\mathrm{E}}$ were low $\left(\mathrm{N}_{\mathrm{E}}\right.$ from the

415 LD method averaged 58.0 across five populations), but were not unusual for LD-derived

416 estimates of $\mathrm{N}_{\mathrm{E}}$ in salamanders. Published estimates of $\mathrm{N}_{\mathrm{E}}$ reported for amphibians have

417 typically been under 100 (Jehle \& Arntzen 2002). Effective population size estimates reported

418 here were similar to values reported for the congeneric endangered California tiger salamander

419 (A. californiense; $\mathrm{N}_{\mathrm{E}}$ values of 11-64; Wang et al. 2011), and for the long-toed salamander ( $A$.

420 macrodactylum; $\mathrm{N}_{\mathrm{E}}=23-207$; Funk et al. 1999). In A. macrodactylum, Savage et al. (2010)

421 estimated $\mathrm{N}_{\mathrm{E}}$ for 47 breeding populations and more than half of these estimates were less than

422 50. Life history factors may provide some explanation for low $\mathrm{N}_{\mathrm{E}}$ in this group as salamanders

423 often exhibit high variance in reproductive success and larval survival within populations.

424 Variance in reproductive success will increase relatedness among individuals in a population,

425 inflating linkage disequilibrium across loci and reducing $\mathrm{N}_{\mathrm{E}}$.

427 be challenging due to the sample sizes required for LD detection. Many threatened taxa are

428 inherently rare and prohibitively difficult to sample in large numbers. For robust estimation of

$429 \mathrm{~N}_{\mathrm{E}}$, it is recommended that sample sizes be greater than 30 for most systems (. When the number

430 of sampled individuals is much smaller than the effective size, LD-based $\mathrm{N}_{\mathrm{E}}$ estimates can be

431 downwardly biased and confidence intervals can be large (or infinite) due to inadequate signal in

432 the dataset (Waples \& Do 2010). We limited our $\mathrm{N}_{\mathrm{E}}$ analysis to populations with sample sizes > 

interval for our $\mathrm{N}_{\mathrm{E}}$ estimate in population Sumner 8 was likely due to an inadequate sample size

435 for this population $(N=21)$.

436

437

438

439

440

441

442

443

444

445

446

447

448

449

450

451

452

453

454

455

\section{Phylogenetic Reconstructions and Population Structure}

Mitochondrial gene-tree reconstructions suggest a complex biogeographic history for $A$. barbouri in Tennessee that involves populations of unisexual Ambystomatids, A. texanum and populations of $A$. barbouri from the northern core region (Kentucky, Indiana, and Ohio). Our results support findings by Bogart et al. (2007) demonstrating a monophyletic relationship of unisexual Ambystomatid mtDNA haplotypes nested within A. barbouri. The phylogenetic reconstruction shown here further details this relationship whereby unisexual Ambystomatids likely shared a maternal common ancestor with $A$. barbouri populations in Kentucky and populations from Middle Tennessee. This pattern, coupled with phylogenies based on nuclear genes, points to a history of repeated hybridization events initiated by an A. barbouri maternal ancestor in the southern portion of its range.

With regards to their mitochondrial lineage, $A$. texanum is nested within present-day $A$. barbouri, rendering A. barbouri paraphyletic. This phylogenetic pattern contradicts the scenario proposed by Kraus and Petranka (1989), that stream-dwelling A. barbouri descended from the more widespread pond-breeding A. texanum. Species-tree reconstructions based on genomewide SNP data are incongruent with relationships recovered from the mitochondrial trees. This species-tree topology adheres to conventional taxonomic and geographical boundaries (Fig. 6), where $A$. texanum is a sister-species to all $A$. barbouri populations. The TMRCA for $A$. barbouri and $A$. texanum based on SNP genotypes was estimated at $\sim 1.6$ million YBP making this a relatively recent split between these ecologically distinct species (Vences et al. 2007). 
456 Incongruence between mitochondrial gene trees and reconstruction based on nuclear data are not

457 uncommon and are often attributed to ancestral lineage sorting, introgression, and/or sex-biased

458 dispersal (Nichols 2001, Toews \& Brelsford 2012). Determining the cause of mitonuclear

459 discordance would require additional sampling across the distributions of both $A$. barbouri and

460 A. texanum; however, it is relevant to note that recent studies have demonstrated that

461 hybridization is common across salamander lineages (including the genus Ambystoma) and may

462 facilitate rapid diversification (Patton et al. 2020).

The hydrogeography of the Central Basin, together with cyclic glacial movements during

464 the Pleistocene, may have shaped contemporary patterns of genetic variation in Tennessee

465 populations of $A$. barbouri. Mitochondrial-based phylogenetic reconstructions and partitioning

466 of genetic variation at SNP loci (including assignment tests, DAPC analyses, AMOVA, and

467 MSC reconstructions) identify the same three genetically distinct groups in Tennessee; these

468 include a northern cluster, a central cluster, and a southern cluster. The Cumberland River

469 appears to have served as a major barrier to gene flow between the northern and the

470 central/southern clusters. The central and southern clusters both occur south of the Cumberland

471 River and are divided by smaller regional drainage patterns. Individuals in the northern and

472 central clusters occupy the Old Hickory Lake and Lower Stones River watersheds, respectively.

473 The central cluster is bordered by the Cumberland River to the North and by the East Fork of the

474 Stones River to the east. The southern cluster includes populations from three watersheds

475 including the West Fork Stones River Watershed, Upper Duck River Watershed, and Mill Creek

476 Watershed; the southern cluster (with the exception of the Wil2 population) is bordered by the

477 Stones River to the north and by the Duck River to the south. 
482 clusters falls within the mid-late Pleistocene. Glacial advances during the Pleistocene did not

483 extend into Kentucky and Tennessee and this region likely served as a refuge for both terrestrial 484 and aquatic fauna (Jacquemin 2016). As glaciers retreated, populations at the northern limits of 485 these refugia would have advanced northward into newly habitable territory. These expansions 486 would have left a genetic signature characterized by reduced genetic variation in the northern 487 end of their distribution as a result of founder effects. Repeated expansions of $A$. barbouri 488 northward from Tennessee may explain the polyphyletic relationships of contemporary

489 Tennessee $A$. barbouri with respect to populations in the north. Specifically, mitochondrial $A$.

490 barbouri populations in northeastern Kentucky, Ohio and Indiana (Clade V) are nested within

491 Tennessee $A$. barbouri as are mitochondrial haplotypes from Ambystomatid unisexuals and $A$.

492 texanum. Genetic evidence of repeated northward expansions has been reported for the Eastern

493 Woodrat (Neotoma floridana; Hayes \& Harrison 1992) and other southeastern fauna (see Hewitt 4941995 for review).

495 Management Implications and Conclusions.

497 management and conservation strategies that may improve the long-term persistence of $A$.

498 barbouri in Tennessee. First, patterns of genetic variation in mitochondrial and nuclear genomes

499 support the assignment of three genetically distinct units for management that warrant the 500 designation of evolutionary significant units (ESUs), where ESUs are defined as groups of 
501 populations that show phylogeographic differentiation for mtDNA haplotypes and divergence in

502 allele frequencies in nuclear markers (Moritz 1994, 1999). These three units include a Northern

503 ESU encompassing all Sumner County populations (S2, S5, S7 and S8), a Central ESU that

504 includes populations from eastern Davidson and Wilson Counties (D3, W1, W3 and W4), and a

505 Southern ESU that includes populations in Williamson, Rutherford, and Bedford Counties

506 (Wil2, B6, R1, R7, and R9). Patterns of differentiation between these three groups of

507 populations suggest a long history of genetic isolation at both mitochondrial and nuclear

508 markers, such that different groups are likely to possess unique combinations of adaptive genetic

509 variation and have likely experienced independent evolutionary trajectories.

511 to genetic diversity and should be ranked highly in regards to conservation priority.

512 Geographically peripheral populations are frequently observed to be genetically less-redundant

513 than more central populations and are more likely to possess potentially adaptive genetic

514 variation (Volkmann et. al. 2014). For A. barbouri, Tennessee populations are geographically

515 disjunct from the majority of A. barbouri breeding populations (Niemiller et al. 2006) and

516 occupy the southernmost edge of the species distribution. Results from this study suggest that

517 Tennessee $A$. barbouri should be prioritized for conservation planning as these populations are

518 both genetically diverse and evolutionarily distinct from populations in the northern part of their

519 distribution. The distinctiveness of these populations is further evidenced by observed

520 differences in reproductive life-history traits, including mean diameter of early stage ovum size

521 and number of eggs per clutch (Niemiller et al. 2009). Prioritizing peripheral populations with

522 adaptive genetic variation and evolutionary potential is even more critical when considering

523 environmental challenges that accompany climate change. Amphibians in general are very 
524 sensitive to climate change as their reproductive life histories are linked to temperature and

525 precipitation (Corn 2005). However, there is evidence that populations at the warm-range edge

526 of their distribution are more resilient (Razgour et al. 2019). Protection of $A$. barbouri breeding

527 sites in Tennessee may be instrumental to ensuring the long-term viability of this species as a

528 whole.

\section{Acknowledgments}

530 We thank Tennessee Wildlife Resources Agency (TWRA) for providing funding to

531 support this work. Ryan Hanscom assisted with sample collections. Joanna Bellan assisted with

532 mitochondrial sequencing and data analysis. Andrea Drayer provided A. barbouri tissue samples

533 from Kentucky populations. We also thank Kristin Womble for producing the map in Figure 2. 


\section{References}

536

537

538

539

540

541

542

543

544

545

546

547

548

549

550

551

552

553

554

555

Allio R, Donega S, Galtier N, Nabholz B. Large variation in the ratio of mitochondrial to nuclear mutation rate across animals: implications for genetic diversity and the use of mitochondrial DNA as a molecular marker. Mol. Biol. Evol. 2017; 34(11):2762-2772.

Anderson MA, Campbell JR, Carey AN, Dodge DR, Johnston RA, Mattison ER, Seddon RJ, Singer NL, Miller BT. Population survey of the streamside salamander in the Nashville Basin of Tennessee. Southeast Nat. 2017: 13(1):101-107.

Toews DP, Brelsford A. The biogeography of mitochondrial and nuclear discordance in animals. Molecular ecology. 2012: 21(16):3907-30.

Bogart JP, Bi K, Fu J, Noble DW, Niedzwiecki, J. Unisexual salamanders (genus Ambystoma) present a new reproductive mode for eukaryotes. Genome 2007; 50(2):119136.

Catchen JM, Amores A, Hohenlohe P, Cresko W, Postlethwait JH. Stacks: building and genotyping loci de novo from short-read sequences. G3-Genes Genom Genet 2011; 1(3):171-182.

Charney ND, Ireland AT, Bettencourt BR. Mapping genotype distributions in the unisexual Ambystoma complex. J Herpetol 2014; 48(2):210-219.

Church SA, Kraus JM, Mitchell JC, Church DR, Taylor DR. Evidence for multiple Pleistocene refugia in the postglacial expansion of the eastern tiger salamander, Ambystoma tigrinum. Evolution 2003; 57(2):372-383.

Corn, P.S., 2005. Climate change and amphibians. Biodiv. Cons. 2005; 28(1). 
Do C, Waples RS, Peel D, Macbeth GM, Tillett BJ, Ovenden JR. NeEstimator v2: re-implementation of software for the estimation of contemporary effective population size (Ne) from genetic data. Mol. Ecol. Resour. 2014; 14(1):209-214.

Eaton DAR. PyRAD: Assembly of de novo RADseq loci for phylogenetic analyses. Bioinformatics 2014; 30(13):1844-1849.

Earl DA, VonHoldt BM. Structure Harvester: a website and program for visualizing STRUCTURE output and implementing the Evanno method. Conserv. Genet. Resour. 2012; 4(2):359-361.

Eastman JM, Niedzwiecki JH, Nadler BP, Storfer A. Duration and consistency of historical selection are correlated with adaptive trait evolution in the streamside salamander, Ambystoma barbouri. Evolution 2009; 63(10):2636-2647. PLoS One. 2011; 6(5):e19379. software STRUCTURE: a simulation study. Mol. Ecol. 2005; 14:2611-2620. data. Genetics 1992; 131(2):479-491.

575 Excoffier L, Lischer HEL. Arlequin suite ver 3.5: a new series of programs to perform 576 population genetics analyses under Linux and Windows. Mol. Ecol. Resour. 2010; 10(3):564-567. 
Funk WC, Tallmon DA, Allendorf FW. Small effective population size in the long-toed

579

580

581

582

583

584

585

586

587

588

589

590

591

592

593

594

595

596

597

598 salamander. Mol Ecol 1999; 8(10):1633-1640.

Fusco, N.A., Pehek, E. and Munshi-South, J. Urbanization reduces gene flow but not genetic diversity of stream salamander populations in the New York City metropolitan area. Evol. App. 2010; 14(1): 99-116.

Geist J. Strategies for the conservation of endangered freshwater pearl mussels (Margaritifera margaritifera L.): a synthesis of conservation genetics and ecology. Hydrobiologia 2010; 644(1):69-88.

Haasl RJ, Payseur BA Multi-locus inference of population structure: a comparison between single nucleotide polymorphisms and microsatellites. Heredity 2011; 106(1):158-171.

Hall TA. BioEdit: a user-friendly biological sequence alignment editor and analysis program for Windows 95/98/NT. Nucl Acid 1999; 41:95-98.

Hare MP. Prospects for nuclear gene phylogeography. Trends Ecol. Evol. 2001; 16(12):700706. https://doi.org/10.1016/S0169-5347(01)02326-6

Hartl DL, Clark AG. Principles of population genetics.1997; Sinauer Assoc. Inc, Sunderland, Massachusetts.

Hayes JP, Harrison RG. Variation in mitochondrial DNA and the biogeographic history of woodrats (Neotoma) of the eastern United States. Systematic Biology. 1992; 41(3):33144.

Heredia-Bobadilla RL, Monroy-Vilchis O, Zarco-González MM, Martínez-Gómez D, Mendoza-Martínez GD, Sunny A (2016) Genetic structure and diversity in an isolated 
600

601

602

603

604

605

606

607

608

609

610

611

612

613

614

615

616

617

618

619

620

621

population of an endemic mole salamander (Ambystoma rivulare Taylor, 1940) of central Mexico. Genetica 2016; 144(6): 689-698.

Hewitt GM. Some genetic consequences of ice ages, and their role in divergence and speciation. Biological journal of the Linnean Society. 1996;58(3):247-76.

Hewitt G. The genetic legacy of the Quaternary ice ages. Nature. 2000; 405(6789):907-13.

Huang H, Knowles, LL. Unforeseen consequences of excluding missing data from nextgeneration sequences: simulation study of RAD sequences. Sys. Bio. 2016; 65(3): 357365.

Huelsenbeck JP, Ronquist F. MRBAYES: Bayesian inference of phylogenetic trees. Bioinformatics 2001; 17(8): 754-755.

Jacquemin SJ, Ebersole JA, Dickinson WC, Ciampaglio CN. Late Pleistocene fishes of the Tennessee River Basin: an analysis of a late Pleistocene freshwater fish fauna from Bell Cave (site ACb-2) in Colbert County, Alabama, USA. PeerJ. 2016; 2(4):e1648.

Jehle R, Arntzen JW. Microsatellite markers in amphibian conservation genetics. Herpetol J $2002 ; 12: 1-9$.

Jombart T, Ahmed I. Adegenet 1.3-1: new tools for the analysis of genome-wide SNP data. Bioinformatics 2011; 27(21): 3070-3071.

Jombart T, Collins C. A tutorial for discriminant analysis of principal components (DAPC) using adegenet 2.0.0. London, Imperial College London, MRC Centre for Outbreak Analysis and Modelling. 2015.

Kardos M, Armstrong E, Fitzpatrick S, Hauser S, Hedrick P, Miller, J., Tallmon, D.A. and Funk, W.C., 2021. The crucial role of genome-wide genetic variation in conservation. 
Keenan K, McGinnity P, Cross TF, Crozier WW, Prodöhl PA. diveRsity: An R package for the estimation of population genetics parameters and their associated errors, Methods in Ecology and Evolution. 2013; 4(8): 782-788.

Kopelman, Naama M., Jonathan Mayzel, Mattias Jakobsson, Noah A. Rosenberg, and Itay Mayrose. "Clumpak: a program for identifying clustering modes and packaging population structure inferences across K." Molecular ecology resources 15, no. 5 (2015): 1179-1191.

Kraus F, Petranka JW. A new sibling species of Ambystoma from the Ohio River drainage. Copeia 1989; 94-110.

Micheletti SJ, Storfer A. An approach for identifying cryptic barriers to gene flow that limit species' geographic ranges. Mol Ecol 2017; 26(2):490-504.

Miller MA, Pfeiffer W, Schwartz T. The CIPRES science gateway: a community resource for phylogenetic analyses. InProceedings of the 2011 TeraGrid Conference: extreme digital discovery 2011; Jul 18: 1-8.

Moritz C. Defining ‘evolutionarily significant units’ for conservation. Trends Ecol. Evol. 1994: 9(10):373-375.

Moritz, C. Conservation units and translocations: strategies for conserving evolutionary processes. Hereditas 1999; 130(3):217-228.

Niedzwiecki JH. Evolutionary history and hybridization of two mole salamander sister species from different habitats. Dissertation, University of Kentucky. 2005

Nichols R. Gene trees and species trees are not the same. Trends in Ecology \& Evolution. $2001 ; 16(7): 358-64$. 
Niemiller ML, Glorioso BM, Nicholas C, Phillips J, Rader J, Reed E, Sykes KL, Todd J, Wyckoff GR, Young EL, Miller BT. Status and distribution of the Streamside Salamander, Ambystoma barbouri, in middle Tennessee. Am. Midl. Nat. 2006;156(2):394-399.

Niemiller ML, Glorioso BM, Nicholas C, Phillips J, Rader J, Reed E, Sykes KL, Todd J, Wyckoff GR, Young EL, Miller BT. Notes on the reproduction of the Streamside Salamander, Ambystoma barbouri, from Rutherford County, Tennessee. Southeast Nat. 2009; 8(1):37-44.

Nunziata SO, Lance SL, Scott DE, Lemmon EM, Weisrock DW. Genomic data detect corresponding signatures of population size change on an ecological time scale in two salamander species. Mol. Ecol. 2017 26(4):1060-1074.

Nunziata SO, Weisrock DW Estimation of contemporary effective population size and population declines using RAD sequence data. Heredity 2018; 120(3): 196-207.

Patton AH, Margres MJ, Epstein B, Eastman J, Harmon LJ, Storfer A. Hybridizing salamanders experience accelerated diversification. Scientific reports. $2020 \mathrm{Apr}$ $16 ; 10(1): 1-2$.

Posada D, Crandall KA. Modeltest: testing the model of DNA substitution. Bioinformatics (Oxford, England) 1998; 14(9):817-818.

Pritchard JK, Wen W, Falush D. Documentation for STRUCTURE software: Version 2. Department of Human Genetics, University of Chicago, Chicago. 2003 Razgour O, Forester B, Taggart JB, Bekaert M, Juste J, Ibáñez C, Puechmaille, SJ, NovellaFernandez R, Alberdi A, Manel S. Considering adaptive genetic variation in climate 
change vulnerability assessment reduces species range loss projections. Proc Nat Acad

667

668

669

670

671

672

673

674

675

676

677

678

679

680

681

682

683

684

685

686

687

Sci 2019.; 116(21):10418-10423.

Reis M and Yang Z. In: Anisimova M (ed.) Evolutionary Genomics. Methods in Molecular Biology, vol 1910. Humana, New York, NY. 2019

Savage WK, Fremier AK, Bradley Shaffer H. Landscape genetics of alpine Sierra Nevada salamanders reveal extreme population subdivision in space and time. Mol. Ecol. 2010 19(16):3301-3314.

Schmidt C, Garroway CJ. The population genetics of urban and rural amphibians in North America. Mol. Ecol. 2021

Scott AF, Miller BT, Brown M, Petranka JW. Geographic distribution: Ambystoma barbouri. Herpetol. Rev. 1997; 28:155.

Shaffer HB, McKnight ML.The polytypic species revisited: genetic differentiation and molecular phylogenetics of the tiger salamander Ambystoma tigrinum (Amphibia: Caudata) complex. Evolution 1996; 50(1):417-433.

Tennessee Wildlife Resources Agency Rules of Biodiversity. Rules and Regulations for in Need of Management, Threatened, and Endangered Species. Chapter 1660-01-32. 2018. Thompson JD, Gibson TJ, Higgins DG. Multiple sequence alignment using ClustalW and ClustalX. Curr. Protoc. Bioinformatics 2003; (1):2-3.

Vences, M. and Wake, D.B., 2007. Speciation, species boundaries and phylogeography of amphibians. Amph. Biol.; 2007: 2613-671.

Volkmann L, Martyn I, Moulton V, Spillner A, Mooers AO (2014) Prioritizing populations for conservation using phylogenetic networks. PLoS One 2014; 9(2):e88945. 
Waples RS, Do C (2008) LDNE: a program for estimating effective population size from data on linkage disequilibrium. Mol. Ecol Res. 2008; 8: 753-756. correlated with breeding pond size in the endangered California tiger salamander, Ambystoma californiense. Conserv. Genet. 2011; 12(4):911-920. Tennessee. 2009

Yang Z. The BPP program for species tree estimation and species delimitation. Current Zoology. 2015; 61(5):854-65. two highly divergent mitochondrial lineages in spotted salamanders (Ambystoma maculatum). Evolution 2003; 57(7):1631-1652. 
701

702

703

704

705

706

707

708

709

710

711

712

713

714

715

716

717

718

719

720

721

722

723

724

725

Table 1. Population IDs, sample sizes for mtDNA/GBS analyses, and map coordinates for all populations.

Population IDs for A. barbouri collections correspond to map locations in Figure 1. For outgroup samples, AAFB denotes Arnold Air Force Base and AKT1 denotes A. texanum from Arkansas. Population IDs by county are as follows: Bedford County (B6), Davidson County (D3), Rutherford County (R1, R7, and R9), Sumner County (S2, S5, S7, and S8), Wilson County (W1, W3, and W4), and Williamson County (Wil2).

Population ID (Map ID) $\begin{aligned} & \text { Sample Size } \\ & \text { mtDNA/GBS Latitude } \quad \text { Longitude }\end{aligned}$
A. barbouri - Tennessee

\begin{tabular}{llll}
\hline Sumner 2 (S2) & $3 /--$ & 36.336487 & -86.502042 \\
\hline Sumner 5 (S5) & $3 / 11$ & 36.329443 & -86.570217 \\
\hline Sumner 7 (S7) & $5 / 37$ & 36.363339 & -86.537930 \\
\hline Sumner 8 (S8) & $6 / 22$ & 36.367510 & -86.591100 \\
\hline Davidson 3 (D3) & $3 / 29$ & 36.100821 & -86.535713 \\
\hline Wilson 1 (W1) & $5 / 15$ & 36.040205 & -86.321052 \\
\hline Wilson 3 (W3) & $4 / 12$ & 36.093415 & -86.372530 \\
\hline Wilson 4 (W4) & $3 / 6$ & 36.093249 & -86.398001 \\
\hline Williamson 2 (Wi12) & $3 / 5$ & 35.950021 & -86.648606 \\
Rutherford 1 (R1) & $3 / 20$ & 35.719718 & -86.353396 \\
Rutherford 7 (R7) & $12 / 28$ & 35.672435 & -86.315247 \\
Rutherford 9 (R9) & $3 / 11$ & 35.752811 & -86.306184 \\
Bedford 6 (B6) & $4 / 8$ & 35.669700 & -86.528800 \\
A. barbouri - & & & \\
Kentucky & & 37.772370 & -84.569400 \\
Kentucky FC (FC) & $3 / 3$ & 37.897710 & -84.393000 \\
Kentucky (RR) & $5 / 4$ & 38.490100 & -85.359400 \\
Kentucky SL (SL) & $4 / 3$ & 38.251190 & -84.753400 \\
Kentucky SW (SW) & $4 / 5$ & 37.960310 & -84.818100 \\
Kentucky JS (JS) & $2 / 2$ & 35.8300 \\
Outgroups & & 35.936860 & -78.567865 \\
A. mabeei & $2 /--$ & 35.392500 & -86.099722 \\
A. maculatum (AAFB) & $2 /--$ & 35.392500 & -86.099722 \\
A. talpoideum (AAFB) & $1 / 1$ & 35.392500 & -86.099722 \\
A. texanum (AAFB) & $--/ 4$ & $35.828812-$ & -90.688313 \\
A. texanum (AKT1) & $1 / 1$ & & \\
\hline
\end{tabular}


Table 2. Primers used for PCR amplification of the mitochondrial D-loop. Primers THR (forward) and 651 (reverse) were used for initial amplification of entire $\sim 1300 \mathrm{bp}$. Internal primers 007 (forward), DL3 (reverse), and DL1

729 (reverse) were used for Sanger sequencing (Shaffer \& McKnight, 1996).

\begin{tabular}{ll}
\hline Primer Name & Sequence $\mathbf{5} \boldsymbol{\rightarrow} \mathbf{3}$, \\
\hline THR (forward) & AACATCGATCTTGTAAGTC \\
007 (forward) & GCACCCAAAGCAAAATTCTTG \\
DL3 (reverse) & TTCGATCCAATTGATGAATG \\
DL1 (reverse) & AATATTGATAATTCAAGCTCCG \\
651 (reverse) & GTAAGATTAGGACCAAATCT \\
\hline
\end{tabular}


Table 3. Standard measures of genetic diversity for 12 populations of Ambystoma barbouri in Tennessee based 586 SNP loci. Summary statistics include the average number of individuals genotyped per locus $(N)$ and the proportion of SNPs that were polymorphic within each population $(P)$. Allelic richness $\left(\mathrm{A}_{\mathrm{R}}\right)$, observed heterozygosity $\left(\mathrm{H}_{\mathrm{O}}\right)$, and expected heterozygosity $\left(\mathrm{H}_{\mathrm{e}}\right)$ were calculated for all loci and again for only those loci that were polymorphic within each population.

\begin{tabular}{lllllllll}
\hline & & \multicolumn{3}{c}{ All Loci } & \multicolumn{4}{c}{ Polymorphic Loci } \\
Population & $\mathbf{N}$ & $\mathbf{P}$ & $\mathbf{A}_{\mathbf{R}}$ & $\mathbf{H}_{\mathbf{O}}$ & $\mathbf{H}_{\mathbf{e}}$ & $\mathbf{A}_{\mathbf{R}}$ & $\mathbf{H}_{\mathbf{0}}$ & $\mathbf{H}_{\mathbf{e}}$ \\
\hline Sumner 5 & 9.58 & 0.108 & 1.042 & 0.018 & 0.020 & 1.460 & 0.170 & 0.154 \\
Sumner 7 & 22.78 & 0.267 & 1.036 & 0.027 & 0.025 & 1.204 & 0.102 & 0.092 \\
Sumner 8 & 12.97 & 0.185 & 1.020 & 0.025 & 0.025 & 1.259 & 0.136 & 0.127 \\
Davidson 3 & 22.02 & 0.192 & 1.041 & 0.018 & 0.016 & 1.243 & 0.094 & 0.083 \\
Wilson 1 & 7.49 & 0.112 & 0.986 & 0.019 & 0.023 & 1.395 & 0.173 & 0.163 \\
Wilson 3 & 7.07 & 0.045 & 1.032 & 0.017 & 0.016 & 1.514 & 0.217 & 0.184 \\
Wilson 4 & 6.79 & 0.089 & 1.049 & 0.019 & 0.020 & 1.602 & 0.210 & 0.220 \\
Williamson 2 & 4.11 & 0.045 & 1.000 & 0.012 & 0.026 & 1.690 & 0.257 & 0.229 \\
Rutherford 1 & 16.71 & 0.092 & 1.026 & 0.009 & 0.008 & 1.303 & 0.096 & 0.091 \\
Rutherford 7 & 23.57 & 0.140 & 1.032 & 0.011 & 0.011 & 1.240 & 0.076 & 0.079 \\
Rutherford 9 & 7.74 & 0.056 & 1.022 & 0.009 & 0.009 & 1.470 & 0.160 & 0.159 \\
Bedford 6 & 8.74 & 0.060 & 1.026 & 0.010 & 0.009 & 1.467 & 0.167 & 0.142 \\
\hline Average Total & 12.46 & 0.116 & 1.026 & 0.016 & 0.017 & 1.404 & 0.155 & 0.144 \\
\hline
\end{tabular}


Table 4. Estimates of effective population sizes $\left(\mathrm{N}_{\mathrm{E}}\right)$ for five populations of Ambystoma barbouri in Tennessee based on the Linkage Disequilibrium (LD) method and the Heterozygosity Excess Method as performed by $\mathrm{N}_{\mathrm{e}}$ Estimator. The $95 \%$ confidence intervals were estimated by the parametric chi-squared method.

\begin{tabular}{llllllll}
\hline & \multicolumn{9}{c}{ Linkage Disequilibrium } & \multicolumn{3}{c}{ Heterozygote Excess } & \\
Population & $\boldsymbol{N}$ & $\boldsymbol{N}_{\boldsymbol{E}}$ & CI Lower & CI Upper & $\boldsymbol{N}_{\boldsymbol{E}}$ & CI Lower & CI Upper \\
\hline Sumner 7 & 35 & 84.5 & 51.5 & 207.2 & 406.4 & 20.7 & $\infty$ \\
Sumner 8 & 21 & 107.7 & 28.8 & $\infty$ & 192.7 & 18.8 & $\infty$ \\
Davidson 3 & 29 & 52.1 & 33.2 & 106.4 & 24.0 & 13.0 & 182.8 \\
Rutherford 1 & 20 & 14.8 & 8.7 & 30.1 & $\infty$ & 14.6 & $\infty$ \\
Rutherford 7 & 28 & 30.7 & 20.9 & 51.6 & $\infty$ & 22.8 & $\infty$ \\
\hline
\end{tabular}

753 
Table 5. Population pairwise $\mathrm{F}_{\mathrm{ST}}$ estimates averaged across 584 SNP loci for 12 populations of Ambystoma barbouri in Tennessee (below diagonal) and significance of $\mathrm{F}_{\mathrm{ST}}$ estimates ( $\mathrm{p}$-values, below diagonal) estimated from nonparametric permutations of SNP genotypes (100 permutations) as performed by the software Arlequin. ** indicates $p<0.001$.

\begin{tabular}{|c|c|c|c|c|c|c|c|c|c|c|c|c|c|}
\hline & Populations & 1 & 2 & 3 & 4 & 5 & 6 & 7 & 8 & 9 & 10 & 11 & 12 \\
\hline 1. & Sumner 5 & --- & & & & & & & & & & & \\
\hline 2. & Sumner 7 & 0.053 & & & & & & & & & & & \\
\hline 3. & Sumner 8 & 0.022 & 0.055 & & & & & & & & & & \\
\hline 4. & Davidson 3 & 0.650 & 0.614 & 0.647 & & & & & & & & & \\
\hline 5. & Wilson 1 & 0.670 & 0.616 & 0.641 & 0.207 & & & & & & & & \\
\hline 6. & Wilson 3 & 0.660 & 0.590 & 0.622 & 0.055 & 0.104 & & & & & & & \\
\hline 7. & Wilson 4 & 0.581 & 0.527 & 0.558 & 0.111 & 0.157 & 0.092 & & & & & & \\
\hline 8. & Williamson 2 & 0.669 & 0.568 & 0.602 & 0.203 & 0.238 & 0.278 & 0.167 & & & & & \\
\hline 9. & Rutherford 1 & 0.725 & 0.638 & 0.698 & 0.288 & 0.408 & 0.399 & 0.320 & 0.031 & & & & \\
\hline 10. & Rutherford 7 & 0.704 & 0.637 & 0.687 & 0.276 & 0.234 & 0.322 & 0.239 & 0.077 & 0.127 & & & \\
\hline 11. & Rutherford 9 & 0.695 & 0.601 & 0.651 & 0.285 & 0.356 & 0.370 & 0.276 & 0.039 & 0.021 & 0.113 & & \\
\hline 12. & Bedford 6 & 0.698 & 0.600 & 0.655 & 0.280 & 0.386 & 0.385 & 0.292 & 0.000 & 0.051 & 0.157 & 0.085 & --- \\
\hline
\end{tabular}


Table 6. Results of hierarchical analyses of molecular variation (AMOVA) for the SNP dataset from 12 populations of $A$. barbouri in Tennessee. Assignment to mitochondrial clades are as follows: Clade II (S5, S7, and S8), Clade IV (D3, W1, W3, and W4), and Clade III (Wil2, R1, R7, R9, B6). Asterisks indicate significance of $\Phi$ statistics based on 1000 permutations in Arlequin.

\begin{tabular}{llllll}
\hline Source of variation & DF & $\begin{array}{l}\text { Sum of } \\
\text { squares }\end{array}$ & $\begin{array}{l}\text { Variance } \\
\text { components }\end{array}$ & $\begin{array}{l}\text { Percentage of } \\
\text { variation }\end{array}$ & Ф-Statistics \\
\hline Among drainages & 2 & 614.5 & 2.30 & 63.60 & $\varphi_{\mathrm{CT}}=0.636^{*}$ \\
$\begin{array}{l}\text { Among populations } \\
\text { within drainages }\end{array}$ & 9 & 31.24 & 0.08 & 2.09 & $\varphi_{\mathrm{SC}}=0.058^{*}$ \\
Within populations & 384 & 476.24 & 1.24 & 44.23 & $\varphi_{\mathrm{ST}}=0.657^{*}$ \\
\hline Total & 395 & $1,121.97$ & 3.62 & & \\
\hline
\end{tabular}


Figure 2. Map of all surveyed populations of $A$. barbouri in Tennessee and Kentucky. County lines are shown with black borders. Shaded regions indicate major watersheds as depicted in the legend. Tennessee populations are colored by ESU assignment where green circles indicate northern ESU populations, yellow circles indicate central ESU populations, and purple circles indicate southern ESU populations.

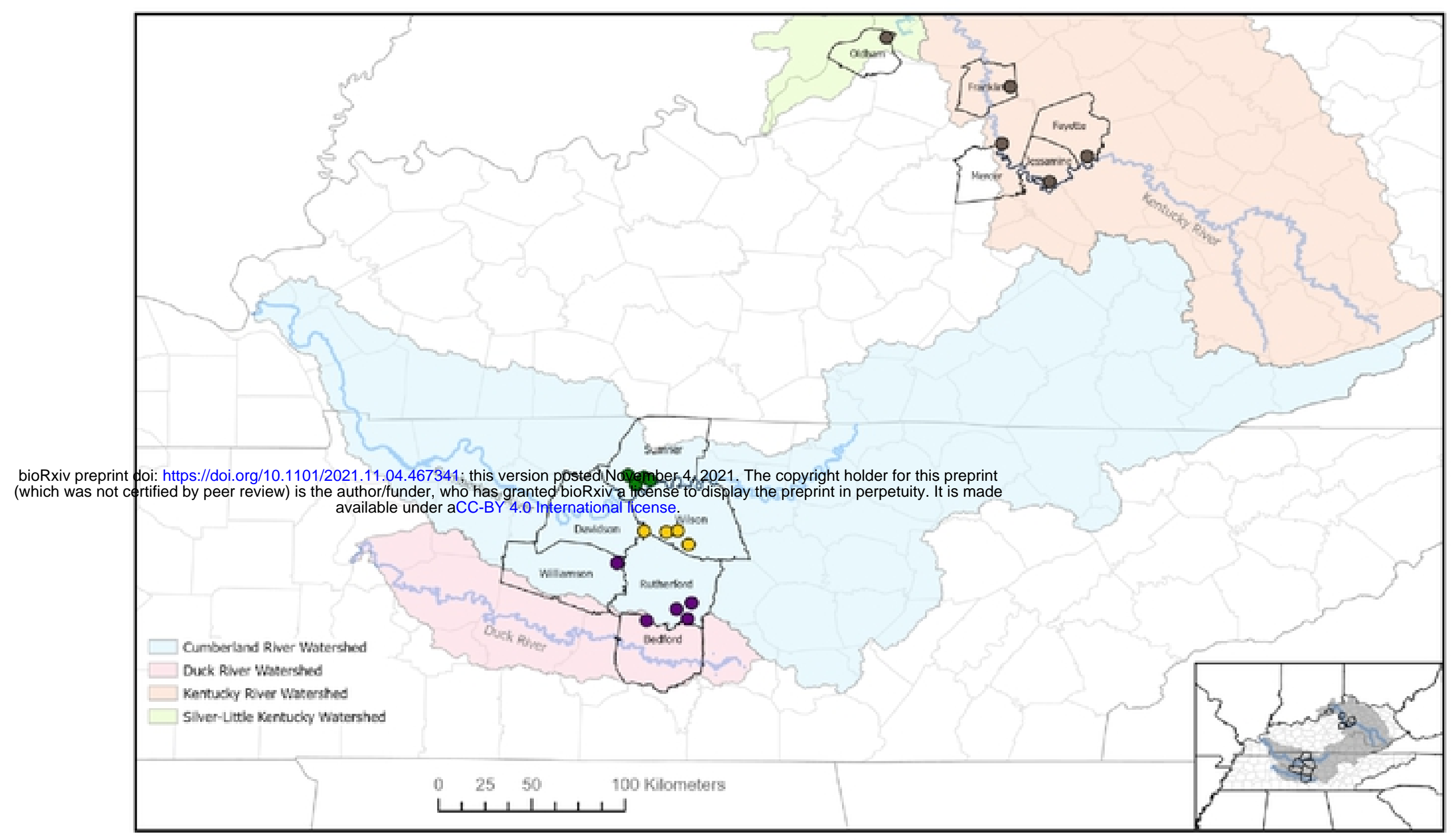

Figure 2 
Figure 4. Discriminant Analysis of Principal Components (DAPC) results based on SNP genotypes A.) DAPC plot for A. barbouri $(N=198)$ from Tennessee. Analysis assumed a value of $\mathrm{K}=3$ as the optimized by BIC analysis. B.) DAPC plot for $A$. barbouri from Tennessee $(\mathrm{N}=198)$ and also including samples from five populations of $A$. barbouri in Kentucky $(N=17)$. Analysis was performed using $\mathrm{K}=3$ as the optimal number of genetic clusters based on BIC analysis. C) DAPC plot for A. barbouri from Tennessee, Kentucky, and also two populations of $A$. texamum $(\mathrm{N}=4)$. Analysis was performed using $\mathrm{K}=5$ as the optimal number of genetic clusters according to the BIC analysis.




Figure 5. Results of Bayesian Assignment tests based on SNPs generated from GBS sequencing. Barplots indicate individual assignment probabilities for samples from 12 A. barbouri populations in Tennessee $(K=2-5)$.



Figure 5 
Figure 6. Species-tree (A) and divergence time estimates (B) from Bayesian multispecies coalescent method as implemented in the software BPP. Numbers at nodes also indicate divergence time estimates ( $t$ ). Divergence time parameter $\tau$ was converted to time in years before present (YBP) in the BPPR statistical package in R-studio.

Shading in tree indicates ESU assignments as follows: Northern ESU (green), Central ESU (Yellow) and Southern ESU (Purple).

(A)




Figure 3. Maximum likelihood phylogenetic reconstructions of unique mitochondrial D-loop haplotypes from Ambystoma barbouri and A. texamum under a GTRCAT model of evolution as performed by RaxML. Bootstrap support values above branches are shown for nodes with $70 \%$ support or greater. Values below nodes indicate posterior probabilities from Bayesian reconstructions under a $\mathrm{T} 92+\mathrm{G}$ model of sequence evolution as performed by MrBayes. Asterisks denote accession numbers for sequences downloaded from Genbank.

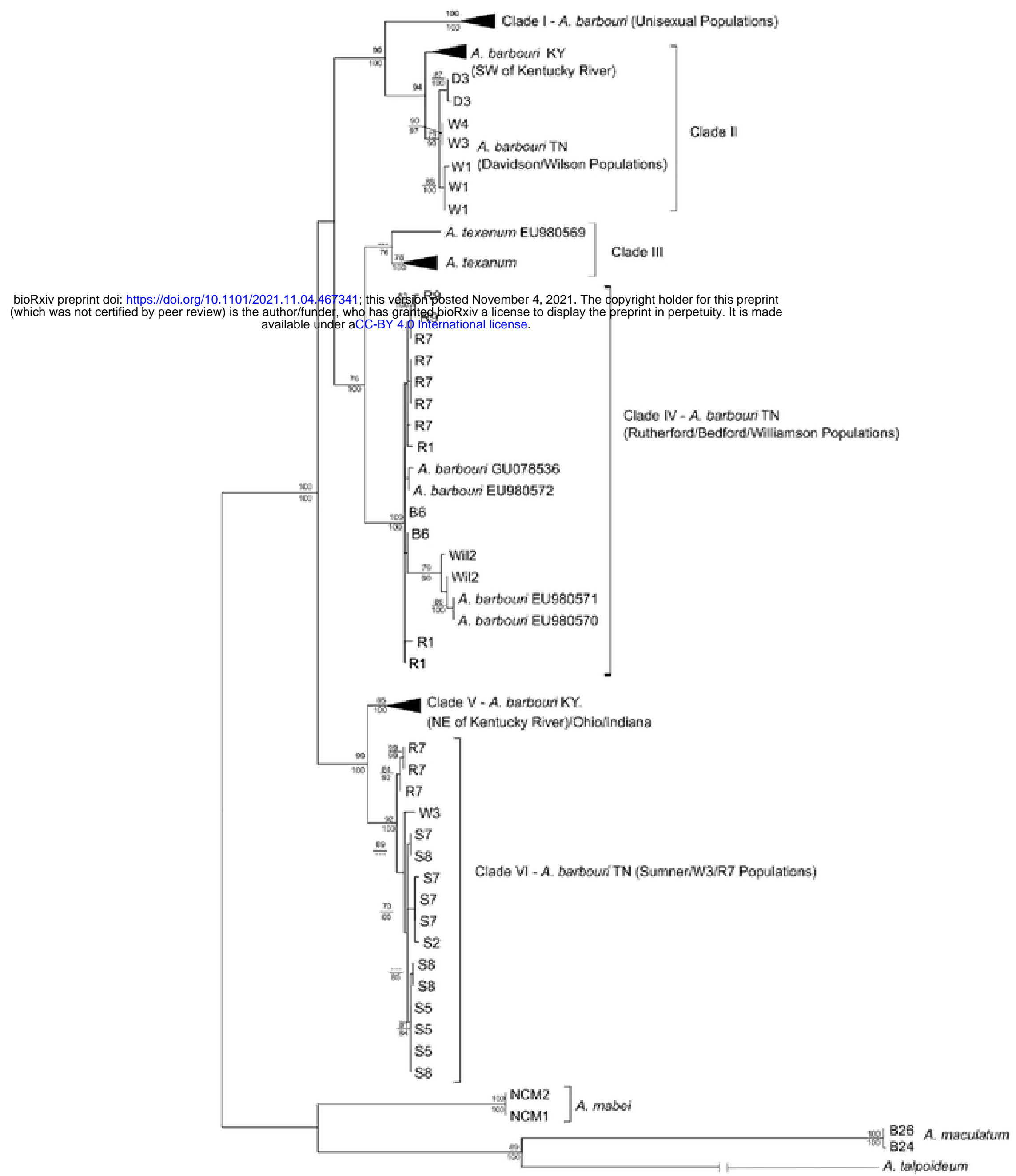

$\operatorname{ata}$

Figure 3 
Figure 1. Photo image of Tennessee Ambystoma barbouri.

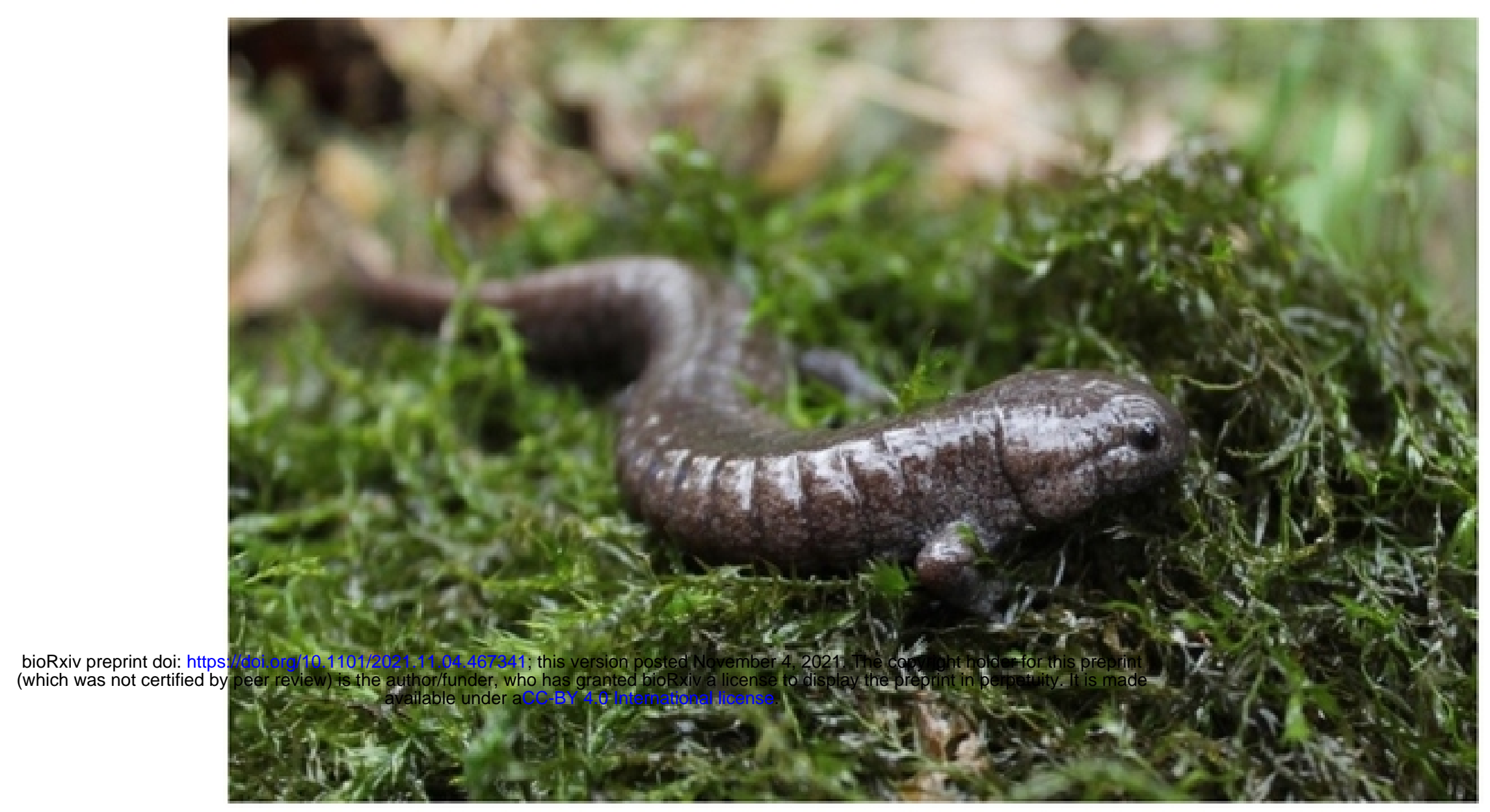

Figure 1 - barb pic 